\title{
A new sensitive detection scheme for helium nanodroplet isolation spectroscopy: application to benzene
}

\author{
Evgeniy Loginov, Andreas Braun and Marcel Drabbels*
}

Received 21st May 2008, Accepted 14th July 2008

First published as an Advance Article on the web 26th August 2008

DOI: $10.1039 / \mathbf{b 8 0 8 2 1 1 a}$

A new method is presented for recording excitation spectra of molecules embedded in helium nanodroplets. The method relies on the complete evaporation of the droplets following excitation of a dissolved molecule and the subsequent detection of the remaining unsolvated molecule by mass spectrometry. The technique has been successfully applied to record the $S_{1}{ }^{1} B_{2 u} \leftarrow S_{0}{ }^{1} A_{1 g}$ transition in benzene. The transition frequencies determined by this new method, beam depletion spectroscopy and REMPI spectroscopy have been found to differ slightly from each other. It is argued that these differences in transition frequency are related to the different droplet sizes probed by the spectroscopic techniques.

\section{Introduction}

During the last decade, helium nanodroplet isolation has established itself as a powerful technique for the spectroscopic investigation of molecular species and their complexes. ${ }^{1-4}$ The low temperature of the helium droplets and the weak interactions of the helium with the solutes allows for the observation of greatly simplified spectra. None of the spectroscopic methods used in helium nanodroplet isolation spectroscopy records directly the absorption spectrum. All methods rely on some kind of action spectroscopy. In case the dissolved molecules fluoresce, the emitted photons can be used as a measure of absorption. However, fluorescence detection is in general limited to electronically excited states and can only be used if the fluorescence is not significantly quenched by the helium environment. Recently, electronically excited states have also been investigated by resonance-enhanced multiphoton ionization (REMPI) spectroscopy. ${ }^{5-9}$ Whereas these two techniques use direct probes of the molecular absorption, almost all other techniques rely on the non-radiative energy transfer from the optically excited molecule to the helium environment. ${ }^{1-4}$ Following this energy transfer, the heated helium droplets cool down to their equilibrium temperature of $0.38 \mathrm{~K}$ by the evaporation of helium atoms from their surface, thereby reducing their size. This reduction in droplet size can be readily detected using mass spectrometry, since the cross section for electron impact ionization of droplets consisting of hundreds or more helium atoms scales with the geometrical cross section, i.e. as $N^{2 / 3}$ with $N$ the number of helium atoms making up the droplet. ${ }^{10}$ Alternatively, a bolometer can be used to measure the energy content of the droplet beam, which is directly related to the droplet size. ${ }^{11}$ Both these spectroscopic techniques are commonly referred to as beam depletion spectroscopy.

Beam depletion spectroscopy is not a background free technique. Instead, it relies on the change of signal due to a change in droplet size. This limits the sensitivity of this

Laboratoire de Chimie Physique Moléculaire, Ecole Polytechnique Fédéral de Lausanne (EPFL), CH-1015 Lausanne, Switzerland method. Nevertheless, by employing a stable helium droplets source in combination with a continuous laser, spectra can be recorded with high signal-to-noise ratios. Recently, Carcabal et al. have demonstrated that it is also feasible to record high quality beam depletion spectra using a high repetition rate pulsed lasers in combination with a multipass excitation arrangement. ${ }^{12}$ Although standard low repetition rate pulsed lasers have also been successfully employed to recorded beam depletion spectra, the quality of the spectra has been found to be much lower due to the inherent low duty cycle. ${ }^{13}$ In order to make effective use of the advantages that low repetition rate pulsed lasers have to offer, the sensitivity of the detection method needs to be significantly enhanced. Here we present a new type of beam depletion spectroscopy that is largely background free and therefore in principle more sensitive than the standard method. We demonstrate the potential of the new method by comparing its sensitivity to that of conventional beam depletion spectroscopy based on electron impact ionization. The comparison is based on spectra we have recorded of the $\mathrm{S}_{1}{ }^{1} \mathrm{~B}_{2 \mathrm{u}} \leftarrow \mathrm{S}_{0}{ }^{1} \mathrm{~A}_{1 \mathrm{~g}}$ transition in benzene. The choice of benzene as the test molecule was prompted by a recent study which revealed that the electronic transitions in benzene recorded by conventional beam depletion spectroscopy are systematically shifted to lower frequencies, up to $1 \mathrm{~cm}^{-1}$, compared to transitions recorded by multiphoton ionization. ${ }^{8}$ In this study, we will readdress this issue by comparing the transition frequencies recorded by the new method as well as beam depletion and REMPI spectroscopy.

\section{Method}

The proposed method relies on the fact that upon absorption of a photon by a molecular chromophore embedded in a helium droplet, the transfer of energy to the helium environment leads to a complete evaporation of the droplet. Following the evaporation of the droplet, the remaining unsolvated molecules are detected. As the new method relies on the complete evaporation of the droplets, we will refer to it as evaporation spectroscopy. 
Although simple in conception, some important issues have to be considered when implementing this method. Firstly, in order to produce bare molecules the energy transferred from the molecule to the helium environment should be sufficient to evaporate the droplet completely. Secondly, the energy transfer and subsequent evaporation of the droplets should be complete during the time delay between the excitation and detection of the molecule. The energy transfer from a chromophore to the helium is known to depend strongly on the type of excitation. It has been established that vibrational excitations of molecules relax very efficiently in helium droplets. ${ }^{1-4}$ Recent experiments have revealed that even extremely vibrationally hot molecules formed by recombination of radicals in helium droplets relax completely on a nanosecond time scale. ${ }^{14}$ Because of this efficient relaxation, the whole energy of the photon becomes available for the evaporation of the droplet. The situation is quite different for electronically excited molecules. Whereas vibrations in electronically excited states relax just as efficiently as in the electronic ground state, the electronic excitation itself relaxes much slower. ${ }^{15}$ This can have a profound effect on the amount of energy deposited into the droplet, especially if the molecule has a large fluorescence quantum yield. Following fluorescence, the vibrational energy content of the molecule is in general substantially lower than the photon energy. Consequently, only a fraction of the photon energy will be transferred to the droplets. In most polyatomic molecules, intersystem crossing (ISC) and internal conversion (IC) compete with fluorescence. For molecules undergoing internal conversion, the whole photon energy will be efficiently transferred to the helium droplet. The situation is less obvious for molecules undergoing intersystem crossing. Clearly, the vibrational excess energy of the metastable electronic state populated will be transferred to the droplets. Whether the electronic energy of this state will be transferred to the droplets has not been established but appears very unlikely. To summarize, depending on the fluorescence, IC and ISC rates of the electronically excited molecule, a fraction or the whole photon energy will be transferred to the helium droplet. When considering the time scale of the experiment, not only the time scale for the relaxation of the energy has to be considered but also that of the evaporation of the atoms from the droplets. The evaporative cooling of helium droplets was first described by Brink and Stringari. ${ }^{16}$ Their calculations showed that most of the atoms evaporate from the droplets on a nanosecond timescale. Consequently, a time delay of several tens to hundreds of nanoseconds between the excitation and the detection of a chromophore will be sufficient to record evaporation spectra.

The maximum droplet size that allows for a complete evaporation of the droplet upon energy transfer from the molecule can be estimated based on the binding energy of a single helium atom to a helium droplet. Although this binding energy is known to be droplet size dependent, to a first approximation it can be assumed to equal the bulk binding energy of $5.0 \mathrm{~cm}^{-1} \cdot{ }^{17}$ As molecules have their electronic excitations typically at about $30000 \mathrm{~cm}^{-1}$ it follows that droplets consisting of up to 6000 helium atoms will potentially completely evaporate. The production of droplets of this size range is readily achieved by today's helium droplet sources. The situation, however, is less favorable when considering vibrational excitations. Vibrational frequencies are typically below $3500 \mathrm{~cm}^{-1}$ and thus require droplets consisting of less than 700 helium atoms. Although droplets of these sizes can be produced, the fluxes attainable are generally much lower than for larger droplets.

For evaporation spectroscopy to work properly, the number of molecules detected that do not result from the evaporation of the helium droplets has to be reduced to a minimum. This implies that the number of molecules present as background gas in the detection chamber has to be minimized. This can be achieved by using differential pumping stages and placing cryoshields in the detection chamber for extra pumping. The ultimate background of gas phase molecules in the detection chamber is in that case determined by the effusive beam of molecules emerging from the doping region. This flux of molecules can be estimated to be at least one order of magnitude smaller than the flux of droplets. Secondly, the ionization scheme used for the detection of the molecules should be insensitive to molecules dissolved in helium droplets. When considering a possible detection scheme, an additional constraint has to be taken into account. Since the number of atoms per helium droplet varies significantly at a given set of experimental conditions, ${ }^{18}$ it is not to be expected that the molecules left after the evaporation of the droplet all reside in the same quantum state. Consequently, the detection scheme should to a large extent be insensitive to the quantum state distribution of the molecules. Direct one-photon ionization just above threshold fulfils these criteria. However, the frequency required for the ionization depends on the molecules under study. Furthermore, for most species these frequencies are not efficiently generated by tabletop laser systems, making this detection scheme not very attractive. Non-resonant ionization by a high intensity ultrashort laser pulse is another potentially useful detection method. In our experiments on the photodissociation of species dissolved in helium droplets ${ }^{14}$ it was found that by adjusting the intensity of the ionizing fsec laser pulse, the formation of ions by direct ionization of molecules in helium droplets can be reduced to a minimum, while still maintaining a high ionization efficiency for unsolvated molecules. This observation is related to the fact that ionization with high intensity fsec laser pulses occurs via tunneling and creates molecular ions with little internal energy. ${ }^{19,20}$ Consequently, the probability that helium droplets will evaporate completely upon ionization to yield bare chromophore ions is small. Based upon the observed signal levels in the photodissociation experiments ${ }^{14}$ we estimate that the probability of yielding chromophore ions is reduced by approximately two orders of magnitude for molecules embedded in helium droplets compared to gas phase molecules. In addition, the ionization efficiency is not expected to be very sensitive to the internal state distribution of the molecules. Non-resonant ionization by ultrashort laser pulses thus fulfils the two prerequisites mentioned above. However, this detection scheme also has a drawback. In order to ionize species non-resonantly, intensities in the range of $10^{14} \mathrm{~W} \mathrm{~cm}^{-2}$ are required. This necessitates tight focusing of the laser beam which, in turn, limits the number of droplets that can be probed. However, if the number of molecules that can be detected in this way is sufficient to achieve good statistics, this tight focusing also presents an advantage, in that the excitation of the chromophore can be limited to this same small volume. When focusing the output of pulsed dye lasers to such small 
volumes, even extremely weak transition, e.g. vibrational overtones, can be used to excite molecules efficiently.

\section{Experimental}

The spectra of the $\mathrm{S}_{1}{ }^{1} \mathrm{~B}_{2 \mathrm{u}} \leftarrow \mathrm{S}_{0}{ }^{1} \mathrm{~A}_{1 \mathrm{~g}}$ electronic transition in benzene are recorded in a helium droplet machine described in detail before. ${ }^{21,22}$ In short, helium droplets are formed by expanding high-purity ${ }^{4} \mathrm{He}$ gas at a pressure of typically 30 bar into vacuum through a $5 \mu \mathrm{m}$ orifice cooled by a closed cycle refrigerator to temperatures in the range of $11-22 \mathrm{~K}$. The droplets created in this way consist on average of 1100 to 20000 helium atoms ${ }^{18,23,24}$ corresponding to droplets with mean radii ranging from 23 to $60 \AA .{ }^{25}$ The helium droplets pick up benzene molecules in a second vacuum chamber, which is flooded with typically $10^{-6}$ mbar of benzene vapor. The doped droplets enter via a differential pumping stage a fourth vacuum chamber which holds two liquid nitrogen cooled shields and an ion imaging setup. At this point, the embedded benzene molecules are electronically excited by UV radiation, having a pulse energy of typically $1 \mathrm{~mJ}$, from a frequency-doubled dye laser (PrecisionScan, Sirah Laser-und Plasmatechnik GmbH) operating at a repetition frequency of $20 \mathrm{~Hz}$. After a time delay of $250 \mathrm{~ns}$ the unsolvated molecules remaining after the evaporation of the helium droplets are non-resonantly ionized by the output of an amplified Ti:Sapphire femtosecond laser system (CPA-1000, Clark-MXR). The output of the femtosecond lasers system, which has a central wavelength of $\lambda=780 \mathrm{~nm}$, a pulse energy of $1.5 \mathrm{~mJ}$ and a pulse duration of $150 \mathrm{fs}$, counter-propagates the dye laser beam and is focused by a $35 \mathrm{~cm}$ focal length lens onto the droplet beam in the center of the ion imaging setup. Here, the created ions are accelerated by the applied electric fields towards position in a sensitive detector. By gating the detector, velocity map images at a specific arrival time of the ions can be recorded. The number of ion impacts in a region of interest, corresponding to a specific velocity distribution, is determined in real time from the images. By monitoring the number of ions as function of excitation frequency, evaporation spectra can be recorded.

By feeding the electrical signal of the detector into a multichannel scaler (P7886, FAST ComTec GmbH), the number of ions created by $1+1$ REMPI and non-resonant ionization can be monitored. In this way, evaporation and REMPI spectra can be recorded simultaneously. The vacuum chamber holding the ion imaging setup also holds a quadrupole mass spectrometer that is mounted with its axis perpendicular to the droplet beam. By sending the laser beam into the setup anti-parallel to the droplet beam it not only passes though the quadrupole mass spectrometer but also the ion imaging setup, thereby making it possible to record simultaneously beam depletion and REMPI spectra.

\section{Results}

We start the evaluation of the new method by presenting in Fig. 1 an overview of the $\mathrm{S}_{1}{ }^{1} \mathrm{~B}_{2 \mathrm{u}} \leftarrow \mathrm{S}_{0}{ }^{1} \mathrm{~A}_{1 \mathrm{~g}}$ excitation spectrum of benzene in helium droplets. The spectrum has been recorded using an integration time of $1 \mathrm{~s}$ at each frequency step of $0.75 \mathrm{~cm}^{-1}$, resulting in a total acquisition

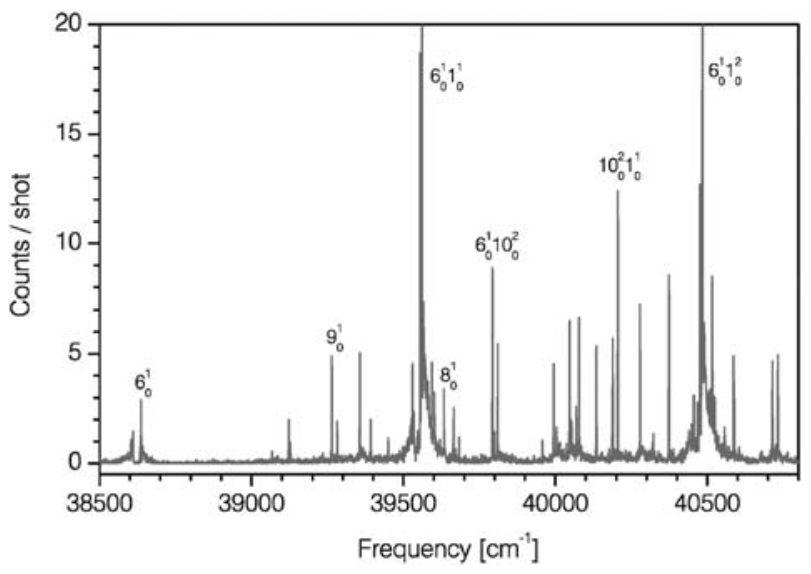

Fig. 1 Overview spectrum of the $\mathrm{S}_{1}{ }^{1} \mathrm{~B}_{2 \mathrm{u}} \leftarrow \mathrm{S}_{0}{ }^{1} \mathrm{~A}_{1 \mathrm{~g}}$ transition in benzene in helium droplets recorded by evaporation spectroscopy. All transitions can be assigned, only the most prominent features have been labeled.

time of $50 \mathrm{~min}$. All of the narrow transitions in the spectrum can be assigned to zero-phonon lines belonging to transition of benzene molecules in helium droplets. In agreement with previous observations, the zero phonon lines are not accompanied by phonon wings. ${ }^{26}$ All the transitions can be readily assigned, as they are on average shifted in frequency by $+30 \mathrm{~cm}^{-1}$ with respect to the corresponding gas phase transitions. $^{27}$ The broad features in the spectrum originate from benzene molecules present in the detection chamber as background gas. The contribution of these benzene molecules to the spectrum has been reduced in several ways. Firstly, the number of molecules in the detection chamber has been minimized by employing differential pumping stages and cryoshields. Secondly, use has been made of the fact that the velocity distribution of background gas benzene molecules is

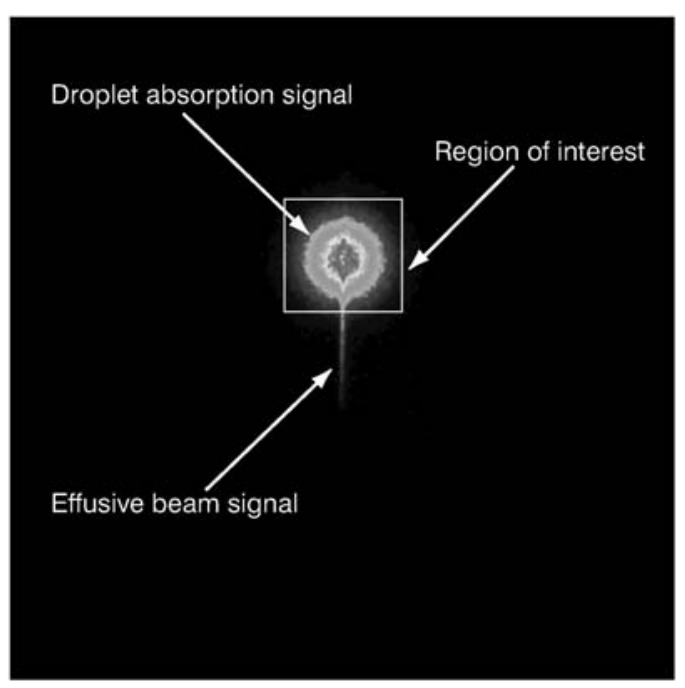

Fig. 2 Ion image recorded by non-resonant ionization of benzene molecules following complete evaporation of helium nanodroplets with a mean radius of $28 \AA$. The embedded benzene molecules have been excited via the $6{ }_{0}^{1} \mathrm{~S}_{1}{ }^{1} \mathrm{~B}_{2 \mathrm{u}} \leftarrow \mathrm{S}_{0}{ }^{1} \mathrm{~A}_{1 \mathrm{~g}}$ transition 250 ns prior to ionization. The square indicates the region of interest used to record the excitation spectra. 


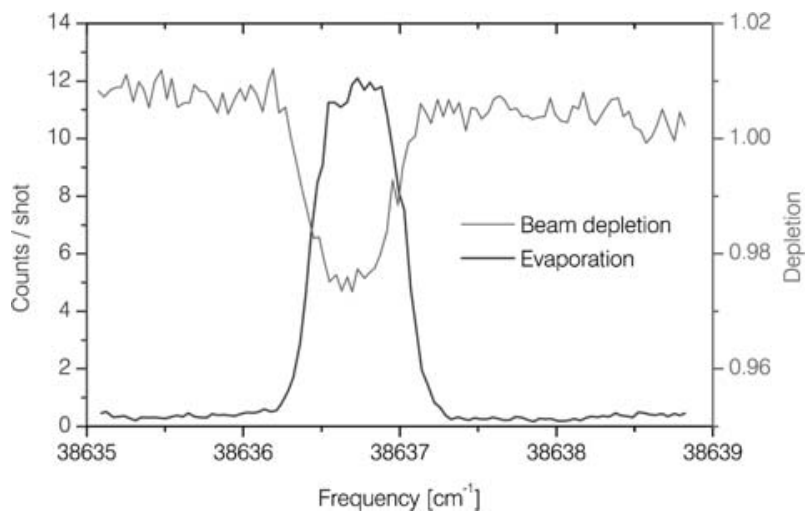

Fig. 3 The $6{ }_{0}^{1} \mathrm{~S}_{1}{ }^{1} \mathrm{~B}_{2 \mathrm{u}} \leftarrow \mathrm{S}_{0}{ }^{1} \mathrm{~A}_{1 \mathrm{~g}}$ transition in benzene embedded in helium droplets with a mean radius of $28 \AA$ recorded by evaporation and beam depletion spectroscopy.

quite different from that of unsolvated molecules remaining after the evaporation of the helium droplets. The velocity distribution of benzene molecules in the effusive beam emerging from the doping region and entering the detection chamber is characterized by a highly anisotropic velocity distribution. The velocity distribution of residual background gas is isotropic and follows a room temperature Maxwell-Boltzmann distribution. In contrast, the benzene molecules resulting from the evaporation of helium droplets are expected to have narrow isotropic velocity distributions. ${ }^{14}$ Fig. 2 shows an ion image recorded at the $6_{0}^{1}$ band of the ${ }^{1} B_{2 u}$ $\leftarrow{ }^{1} \mathrm{~A}_{1 \mathrm{~g}}$ transition of benzene in helium droplets. The image clearly shows two contributions. The weak vertical streak in the ion image corresponds to molecules in the effusive beam. The strong isotropic signal originates from benzene molecules formed by the evaporation of helium droplets. This assignment is based upon the fact that this signal is displaced with respect to the center of the image, as is expected for molecules moving at the speed of the droplet beam. In addition, the speed distribution derived from the image can be fitted to a Maxwell-Boltzmann distribution corresponding to a temperature of $41 \mathrm{~K}$. Although the contribution of residual gas molecules is not easily recognized from the image, analysis of the corresponding speed distribution reveals that residual gas contributes $10 \%$ to the total ion signal. To minimize the contributions from gas phase molecules to the spectrum, only those ions are counted that impinge onto the detector within a region of interest corresponding to the benzene molecules resulting from the evaporation of helium droplets. By taking these measures, the background level in the spectra can be reduced by an order of magnitude and spectra with good signal-to-noise ratios can be obtained. To evaluate the sensitivity of the new method we have also recorded beam depletion spectra. Fig. 3 shows in detail the $6_{0}^{1}$ band of the ${ }^{1} \mathrm{~B}_{2 \mathrm{u}} \leftarrow{ }^{1} \mathrm{~A}_{1 \mathrm{~g}}$ transition of benzene embedded in helium droplets with a mean radius of $28 \AA$. The spectrum has been recorded using an integration time of $5 \mathrm{~s}$ at each frequency step of $0.03 \mathrm{~cm}^{-1}$. The observed transition frequency agrees within the absolute accuracy of $1 \mathrm{~cm}^{-1}$ of our laser to the values reported in the literature. ${ }^{8,26}$ This spectrum should be compared to the beam depletion spectrum, also shown in Fig. 3, that has been recorded under identical conditions but with a 10 times longer

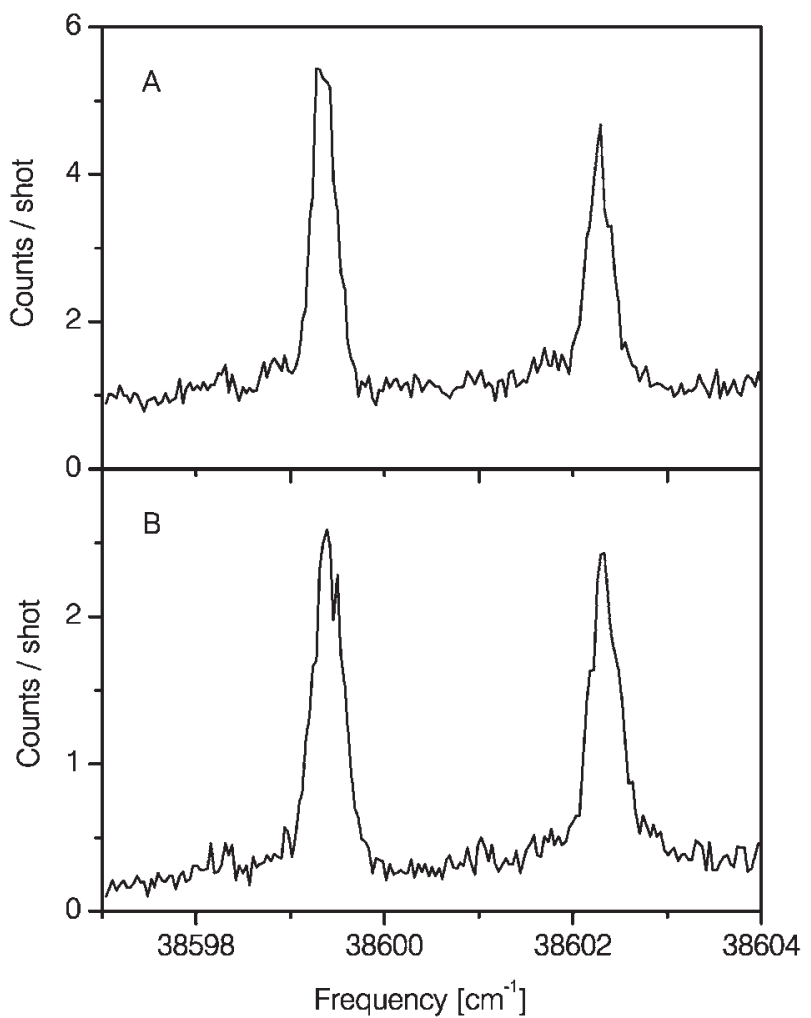

Fig. 4 Spectrum of the $6_{0}^{1} \mathrm{~S}_{1}{ }^{1} \mathrm{~B}_{2 \mathrm{u}} \leftarrow \mathrm{S}_{0}{ }^{1} \mathrm{~A}_{1 \mathrm{~g}}$ transition of the benzene dimer embedded in helium with a mean radius of $28 \AA$. The spectrum of panel A has been recorded by monitoring the benzene ion while that of panel B has been recorded monitoring the (benzene) $)_{2}$ ion.

integration time. Clearly, the signal-to-noise ratio in the evaporation spectrum is much higher, even though the integration time was much shorter. Analysis of the signal and noise levels reveals that the new method is a factor of 250 more sensitive than the standard beam depletion method. It is also worthwhile to compare the signal-to-noise ratio of the evaporation spectra of benzene with the beam depletion spectra as recorded by Schmied et al. using a high repetition rate laser system and bolometric detection. ${ }^{26}$ Inspection of the spectra presented in Fig. 2 of ref. 26 reveals that the signal-to-noise ratio is only slightly less than that obtained with evaporation spectroscopy in the present study. Although this observation might seem surprising at first, it is not if one takes into account the difference in duty cycle between the two experiments. Whereas we estimate that the duty cycle of our experiment is approximately $3 \times 10^{-6}$, Schmied et al. report a value of $5 \%$. Thus, the lower sensitivity of the beam depletion method is almost fully compensated by the much higher duty cycle of the experiment of Schmied et al.

Molecular complexes can be readily formed in helium droplets by doping the droplets with multiple molecules. ${ }^{1-4}$ As the number of molecules picked up by the helium droplets follows a Poisson distribution, it can be difficult at times to assign features in excitation spectra to a specific complex. Having mass information would be of great benefit in the assignment of the spectral features. The new method has the potential to obtain simultaneously spectroscopic and mass information. To explore this potential we have recorded the 


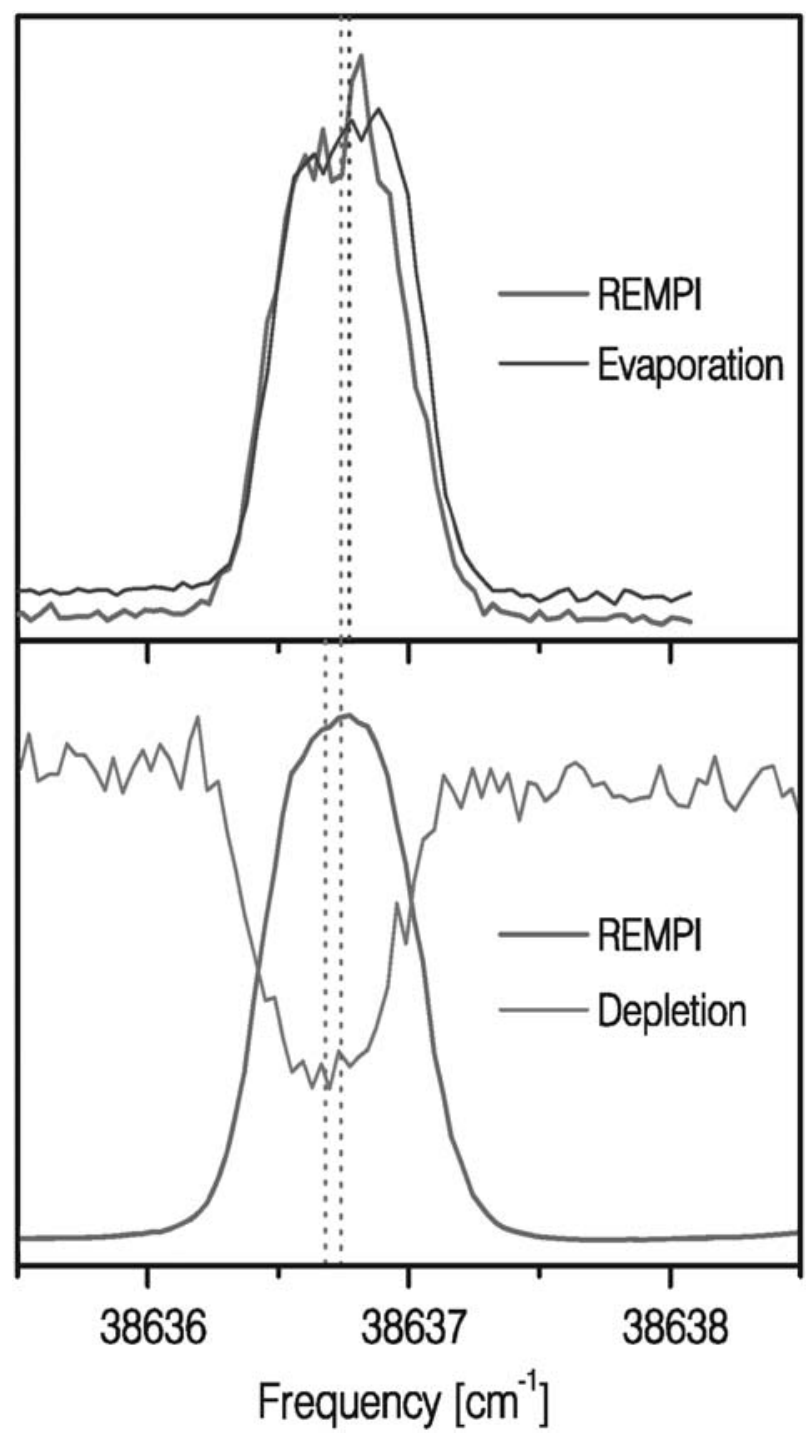

Fig. 5 The $6{ }_{0}^{1} \mathrm{~S}_{1}{ }^{1} \mathrm{~B}_{2 \mathrm{u}} \leftarrow \mathrm{S}_{0}{ }^{1} \mathrm{~A}_{1 \mathrm{~g}}$ transition of benzene embedded in helium droplets with a mean radius of $28 \AA$ recorded by various methods. The spectra in the upper panel are recorded simultaneously by REMPI and evaporation spectroscopy while the spectra in the lower panel are recorded simultaneously by REMPI and beam depletion spectroscopy. The vertical dotted lines indicate the central frequencies of the transitions.

$6_{0}^{1}$ transition of the benzene dimer in helium droplets by gating the detector at the arrival times of benzene and (benzene) $)_{2}$ ions. The resulting spectra are presented in Fig. 4. The upper panel shows the spectrum recorded at the monomer mass. Due to the higher gas pressure used for doping the droplets with multiple species the background level in this spectrum is considerably higher than in that of the benzene monomer shown in Fig. 3. The observed transition frequencies agree well with the values found before by Lehmann and coworkers. ${ }^{26}$ The lower panel of Fig. 4 shows the spectrum recorded at the benzene dimer mass. Although the signal level is a factor of two lower as compared to monomer detection, the signalto-noise ratio is similar due to the lower background. The fact that we observe this spectrum implies that a large fraction of the benzene dimers survives the electronic excitation,

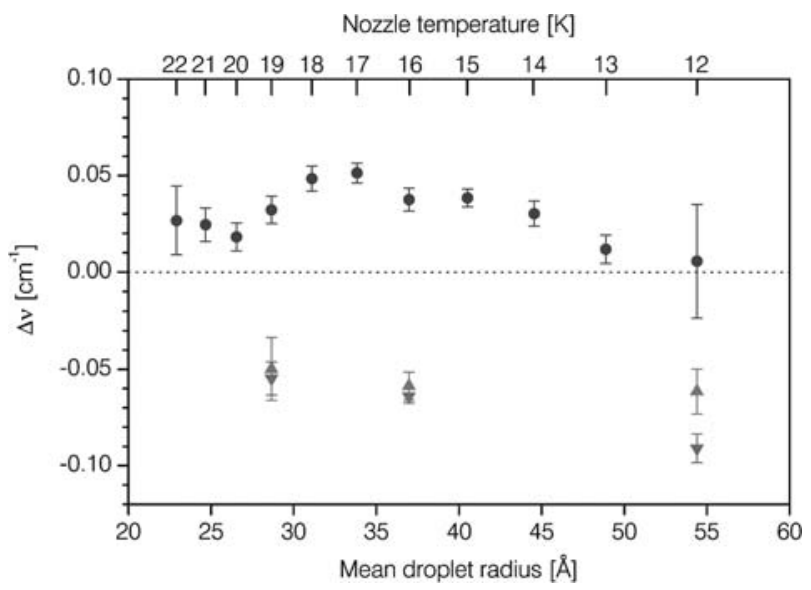

Fig. 6 Central frequency of the $6_{0}^{1}$ benzene transition in helium droplets determined by beam depletion $(\boldsymbol{\nabla})$ and evaporation spectroscopy (-) as function of the mean droplet radius. The reported frequencies are relative to that determined by REMPI spectroscopy. Also shown is the relative transition frequency for the $6_{0}^{1} 1_{0}^{1}$ transition determined by beam depletion spectroscopy ( $\mathbf{A})$.

subsequent relaxation and complete evaporation of the helium droplets. In addition, it shows that non-resonant ionization does not lead to a massive fragmentation of weakly bound complexes, in agreement with previous observations. ${ }^{28}$ Thus the new method, besides being more sensitive than beam depletion spectroscopy, has the additional advantage of providing mass information.

As already mentioned in the Introduction, the use of benzene as a test molecule was prompted by experiments of Stace and coworkers on this molecule. ${ }^{8}$ They observed that the transition frequencies of benzene in helium droplets depends on the detection method employed. More specifically, they found that spectra recorded by beam depletion spectroscopy were systematically shifted to lower frequencies, up to $1 \mathrm{~cm}^{-1}$, compared to those recorded by REMPI spectroscopy. Inspection of the two spectra in Fig. 3 reveals that the beam depletion spectrum is shifted to the red by $0.05 \mathrm{~cm}^{-1}$ with respect to the evaporation spectrum. As these spectra are not recorded simultaneously and the difference is smaller than the resetability of the laser, this small shift could be related to experimental uncertainty in the determination of the laser frequency. In order to minimize this experimental uncertainty we have recorded simultaneously evaporation and REMPI spectra as well as beam depletion and REMPI spectra. Fig. 5 shows the $6_{0}^{1}$ transition of benzene in helium droplets recorded with the various methods. The transitions are clearly shifted with respect to each other. The highest transition frequency is observed for the evaporation spectrum while the lowest frequency is found for the beam depletion spectrum. Spectra of this kind have been recorded for a range of droplet sizes. The differences between the peak positions have been determined by fitting the transition lines to a Gaussian function. The shift of the transition frequencies relative to those measured by REMPI spectroscopy are presented in Fig. 6. The transition frequencies are systematically the highest for the evaporation spectra while those of the beam depletion are always the lowest. The droplet size averaged shifts amount 


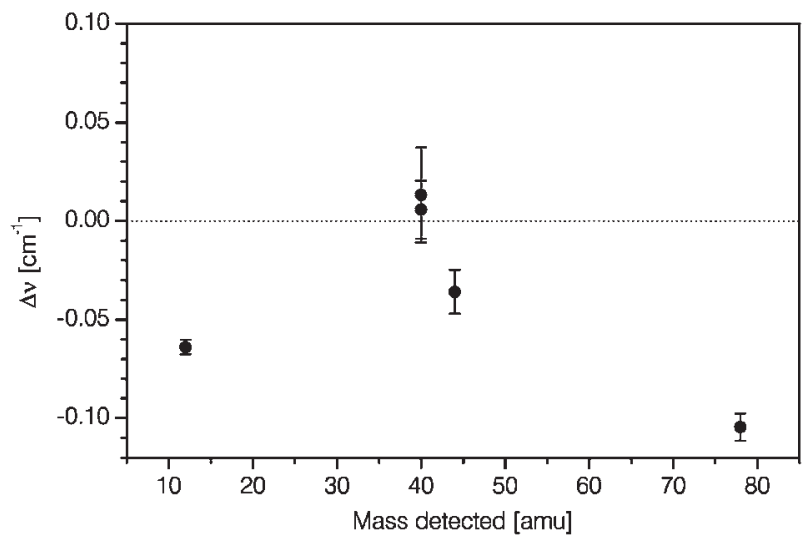

Fig. 7 Central frequency of the $6_{0}^{1} \mathrm{~S}_{1}{ }^{1} \mathrm{~B}_{2 \mathrm{u}} \leftarrow \mathrm{S}_{0}{ }^{1} \mathrm{~A}_{1 \mathrm{~g}}$ transition of benzene in helium droplets with a mean radius of $35 \AA$ as a function of ion fragment detected by beam depletion spectroscopy. The frequencies are given relative to that determined by REMPI spectroscopy.

to $0.035 \pm 0.004 \mathrm{~cm}^{-1}$ and $-0.063 \pm 0.004 \mathrm{~cm}^{-1}$ for the evaporation and beam depletion spectra, respectively. Although these shifts are comparable to the linewidth of the laser, we are confident that these shifts are significant in view of the way these spectra have been obtained. In addition, the shift between the REMPI and beam depletion data is comparable with the results found by Curtis et al. for this transition. ${ }^{8}$ It should be noted that the beam depletion spectra used in this analysis have been recorded by monitoring the ion signal at mass $12 \mathrm{amu}$, corresponding to $\mathrm{He}_{3}{ }^{+}$. As electron impact ionization yields a wide mass distribution of ions, it is very well possible that the spectra are not truly representative of the absorption spectrum of benzene in helium droplets. To address this issue we have recorded additional beam depletion spectra by monitoring masses of 40, 44 and 78, corresponding to $\mathrm{He}_{10}{ }^{+}, \mathrm{He}_{11}{ }^{+}$and $\mathrm{C}_{6} \mathrm{H}_{6}{ }^{+}$, respectively. As can be seen in Fig. 7, the shifts relative to the REMPI spectra do indeed depend on the mass monitored. Whereas the spectra recorded by monitoring masses of 12,44 and 78 reveal a variable but

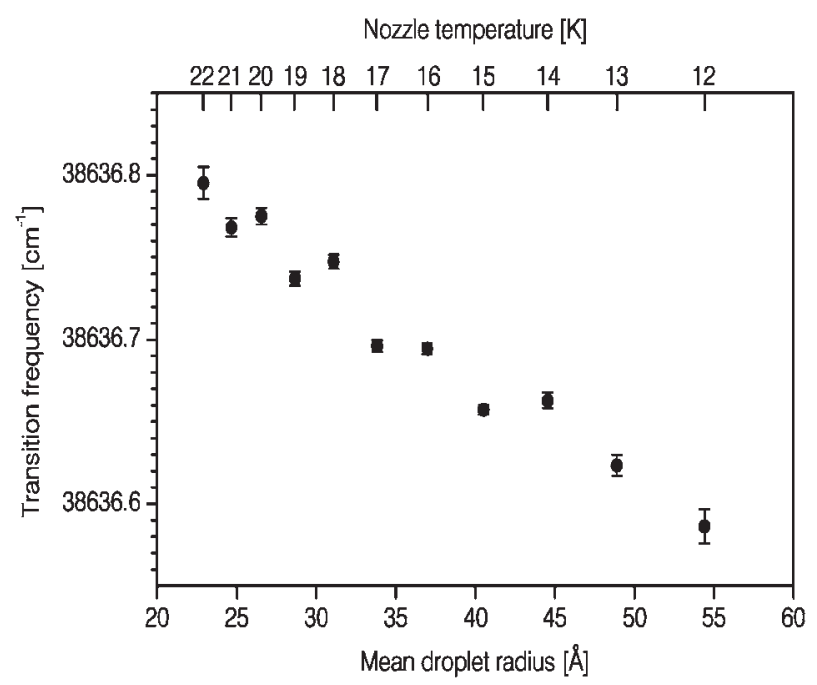

Fig. 8 Variation of the $6{ }_{0}^{1} \mathrm{~S}_{1}{ }^{1} \mathrm{~B}_{2 \mathrm{u}} \leftarrow \mathrm{S}_{0}{ }^{1} \mathrm{~A}_{1 \mathrm{~g}}$ transition frequency in benzene as a function of source temperature, as determined by evaporation spectroscopy. negative shift, the spectrum recorded at a mass of 40 shows a slightly positive shift. It is important to note that the magnitude of these shifts is comparable to that found for the different spectroscopic methods.

In their work, Curtis et al. found that the shift between the REMPI and beam depletion spectrum of the $6_{0}^{1} 1_{0}^{1}$ transition in benzene was by $1 \mathrm{~cm}^{-1}$ substantially larger than that for the $6_{0}^{1}$ transition. ${ }^{8}$ We have also measured this transition in benzene and found a much smaller shift, similar to that for the $6_{0}^{1}$ transition, see Fig. 6. By recording simultaneously the spectra with different methods, the experimental uncertainty in the determination of frequency shifts has been reduced to a minimum. We are therefore convinced that our measurement of the shift is correct. In their paper, Curtis et al. do not specify at which ionic fragment mass their beam depletion spectra have been recorded. However, in view of the relative small variation in the transition frequency of the $6_{0}^{1}$ band with ion mass, we do not expect that the large difference between our results and those of Curtis et al. for the $6{ }_{0}^{1} 1_{0}^{1}$ transition is related to the mass monitored. Most likely, the large discrepancy is related to the uncertainty in determination of the laser frequencies by Curtis et al. since the Burleigh wavemeter used to calibrate their laser is known to indicate at times frequencies deviating by $1 \mathrm{~cm}^{-1}$ from the actual frequency.

In order to be able to discuss the possible origin of the observed shifts, it is important to compare the magnitude of these shifts with respect to the variation of the transition frequency with droplet size. Fig. 8 shows the variation of the central frequency of the $6_{0}^{1}$ transition in benzene with a droplet size as recorded by evaporation spectroscopy. The central frequency decreases monotonously with the droplet radius. The variation of the central frequency over the full range of droplet sizes used in this study amounts to $0.2 \mathrm{~cm}^{-1}$. This variation is noticeably larger than the shifts observed between the different detection methods, which could be an indication that droplet size effects are at the origin of these shifts.

\section{Discussion}

The application of evaporation spectroscopy for the study of electronically excited states has been shown to be straightforward. The spectrum of the weak $\mathrm{S}_{1}{ }^{1} \mathrm{~B}_{2 \mathrm{u}} \leftarrow \mathrm{S}_{0}{ }^{1} \mathrm{~A}_{1 \mathrm{~g}}$ transition in benzene could be recorded with good signal-to-noise ratio within a short time interval. Comparison of this spectrum with that recorded by the standard beam depletion method revealed that evaporation spectroscopy is considerably more sensitive when using pulsed lasers for excitation. The sensitivity of the evaporation method is mainly determined by the flux of doped helium droplets within the detection volume. This flux, and thus sensitivity, can be greatly enhanced by utilizing one of the recently developed pulsed helium droplet sources. ${ }^{29,30}$ However, it should be realized that the ultimate sensitivity when using these pulsed sources will be determined by the background signal caused by the direct non-resonant ionization of molecules in helium droplets by the femtosecond laser pulse. Besides being more sensitive, the new technique has an additional advantage, i.e. it offers the possibility to obtain simultaneously spectral and mass information. This can be useful when trying to disentangle complex spectra originating from multiple species present in the droplets. 
The spectra recorded by the different techniques revealed small differences in transition frequency. Since these differences are smaller than the variation of the transition frequency with droplet size, they possibly result from differences in droplet sizes probed by the three techniques. As already outlined before, several energy transfer processes have to be considered when dealing with electronically excited states. The fluorescence quantum yield of the vibrationless and the $6^{1}$ level in the ${ }^{1} \mathrm{~B}_{2 \mathrm{u}}$ state have been determined to be 0.22 and 0.27 , respectively. ${ }^{31}$ It is generally accepted that intersystem crossing to the $\mathrm{T}_{1}{ }^{3} \mathrm{~B}_{1 \mathrm{u}}$ is the main cause for the low fluorescence quantum yield. This lowest triplet state is located $3.67 \mathrm{eV}$ above electronic ground state. ${ }^{32}$ Consequently, excitation of the $6^{1}{ }^{1} \mathrm{~B}_{2 \mathrm{u}}$ level produces a significant fraction of molecules in the $\mathrm{T}_{1}{ }^{3} \mathrm{~B}_{1 \mathrm{u}}$ state with approximately $9000 \mathrm{~cm}^{-1}$ of vibrational energy. Relaxation of this energy into the helium droplet will lead to the evaporation of about 1800 helium atoms. Accordingly, helium droplets consisting of less than this number of helium atoms will yield bare benzene molecules upon electronic excitation of the embedded molecule. Droplets consisting of more helium atoms will survive the evaporation process, but will shrink significantly in size. These shrunken droplets will contain a benzene molecule in the ground vibrational level of the $\mathrm{T}_{1}{ }^{3} \mathrm{~B}_{1 \mathrm{u}}$ state. Since the lifetime of this state has been found to be $20 \mathrm{~s}$ in $2 \mathrm{~K}$ solid hydrogen matrices, ${ }^{33}$ it is not to be expected that these molecules will relax further in helium droplets on the time scale of the experiment.

When determining the droplet size dependence of REMPI spectroscopy, two energy relaxation processes have to be taken into account. The first process is the relaxation of the benzene molecule following excitation to the $\mathrm{S}_{1}$ state. Experiments on the photoionization of aniline in helium droplets using $1+1$ REMPI have revealed that vibrational relaxation in the $S_{1}$ state is complete within the pulse duration of the laser. ${ }^{5}$ This implies that all vibrational excess energy will be deposited into the droplets before the molecule is ionized. As mentioned above, excitation of benzene to the $S_{1}$ state produces a significant fraction of molecules in the $\mathrm{T}_{1}{ }^{3} \mathrm{~B}_{1 \mathrm{u}}$ state with approximately $9000 \mathrm{~cm}^{-1}$ of vibrational energy. This energy will be transferred to the droplet within the laser pulse. However, molecules that have relaxed to the vibrational ground state of the $\mathrm{T}_{1}{ }^{3} \mathrm{~B}_{1 \mathrm{u}}$ state are not efficiently probed by the REMPI process since absorption of an additional UV photon of $\sim 39000 \mathrm{~cm}^{-1}$ is not sufficient to ionize these benzene molecules. ${ }^{32,34}$ Two-photon ionization of ground state $T_{1}{ }^{3} B_{1 u}$ benzene is in principle possible but the efficiency is expected to be low. Photoelectron spectroscopy on the benzene derivative aniline embedded in helium droplets has shown that ionization via the triplet state does not occur. ${ }^{5}$ Consequently, only those molecules that have relaxed to the vibrationless level of the $S_{1}$ state will be probed by REMPI. The ionization process will populate several vibrational levels in the benzene ion. This vibrational energy will also be transferred to the helium droplet and will lead to the evaporation of additional helium atoms. Based upon the photon energy of $38636 \mathrm{~cm}^{-1}$ and the ionization threshold, the maximum amount of available energy can be estimated. Photoelectron spectroscopy of aniline in helium droplets revealed that the vertical ionization threshold of this benzene derivative is lowered by approximately $800 \mathrm{~cm}^{-1}$ in helium droplets due to polarization of the medium. ${ }^{5}$ Given the benzene ionization threshold of $74556.6 \mathrm{~cm}^{-134}$ and the lowering of this threshold in helium droplets, the maximum energy to be transferred to the droplets can be estimated to be about $3500 \mathrm{~cm}^{-1}$. This will result in the evaporation of approximately 700 helium atoms. Based upon this analysis, it appears that considerably smaller droplets are probed by REMPI than by evaporation spectroscopy. In contrast to both evaporation and REMPI spectroscopy, beam depletion spectroscopy based upon electron impact ionization is not limited to a certain droplet size. However, it should be realized that beam depletion spectroscopy is more sensitive for small than for large droplets since the ionization efficiency scales as $N^{2 / 3}$, with $N$ being the number of helium atoms making up the droplet. In addition, fragmentation patterns following electron impact ionization depend to some extent on droplet size ${ }^{35,36}$ which should be considered when recording spectra by monitoring specific ion masses.

The frequency of the $60_{0}^{1} \mathrm{~S}_{1}{ }^{1} \mathrm{~B}_{2 \mathrm{u}} \leftarrow \mathrm{S}_{0}{ }^{1} \mathrm{~A}_{1 \mathrm{~g}}$ transition was found to decrease as a function of the mean helium droplet radius. This variation with droplet size can be rationalized by the difference in long-range interaction of ground and excited state benzene molecules with the helium. ${ }^{37,38}$ Since the three detection methods used in this study have different droplet size dependent detection efficiencies, one expects this to be reflected in the observed transition frequencies. Indeed, the method that is anticipated to be most sensitive for large droplets, i.e. beam depletion spectroscopy, shows the lowest transition frequency. The average shift of $\sim 0.06 \mathrm{~cm}^{-1}$ is within the variation of the transitions frequency over the full range of mean droplet size used in this study. Furthermore, the variation of the transition frequency with ion fragment is also well within this range. In contrast, REMPI spectroscopy that is expected to be most sensitive to smallest droplets does not show the highest transition frequencies. It should be realized that several assumptions have been made when discussing the droplet size dependence in REMPI spectroscopy. Firstly, when discussing the lowering of the ionization threshold we considered the vertical threshold. It is, however, more appropriate to use the adiabatic threshold, which is expected to be lower in energy. To our knowledge, no estimates of the adiabatic ionization thresholds for molecules in helium have been reported in the literature. Based upon estimates of solvation energies of atomic cations the difference between the two thresholds is not expected to exceed $3000 \mathrm{~cm}^{-1}{ }^{39}$ Even with this additional energy release into the helium droplets, the experimental observations cannot be explained. Secondly, it was assumed that helium atoms evaporate from the droplets statistically after the droplets have reached thermal equilibrium. However, there is increasing experimental ${ }^{40,41}$ as well as theoretical ${ }^{42}$ evidence that the evaporation of helium atoms from droplets following ionization of dissolved species is not statistical, but rather is an explosive-like process. In addition, simulations of the dynamics of ionized neon clusters in helium droplets show that ions are ejected from the droplets in this process. ${ }^{42} \mathrm{By}$ this process, the ejected ions could originate from larger droplets than anticipated, especially if the neutral species are located 
near the surface region of the droplet. Calculations by Lehmann $^{43}$ show that neutral impurities are indeed substantially delocalized within helium droplets. In view of this, the unexpected shift in transition frequency when using REMPI detection is likely to be related to the cooling mechanism of ions in helium droplets.

In conclusion, we argue that the small differences in observed transition frequency are related to the variation of the transition frequency with droplet size in combination with the fact that the methods used probe different droplet sizes. We therefore see no need to invoke a model in which benzene molecules are located at two equilibrium sites in helium droplets as was suggested by Curtis et al. to explain their observations. $^{8}$

\section{Conclusion}

We have presented a new method for recording spectra of molecules dissolved in helium nanodroplets. The new method relies on the complete evaporation of the droplets following excitation of the embedded chromophore. The unsolvated molecules remaining after the evaporation of the droplets are detected following non-resonant ionization by an ultrashort IR laser pulse. As the method is almost background free, the sensitivity has been found to be considerably higher than the standard beam depletion technique based upon electron impact ionization. Furthermore, the new method also provides mass information, which is potentially very valuable for the analysis of complex spectra originating from different species in helium droplets. The technique has been successfully applied to record the $\mathrm{S}_{1}{ }^{1} \mathrm{~B}_{2 \mathrm{u}} \leftarrow \mathrm{S}_{0}{ }^{1} \mathrm{~A}_{1 \mathrm{~g}}$ transition in benzene. A comparison of the spectra recorded with the new method, beam depletion and REMPI spectroscopy reveals that the transition frequencies as determined by the three methods are slightly different. It is argued that this variation in transition frequency is related to the droplet size dependence of the transition frequency and the difference in droplet sizes probed by the various methods.

\section{Acknowledgements}

This research was made possible by the financial support of the Swiss National Science Foundation through Grant Nos. 200020-112193 and 200020-119789.

\section{References}

1 J. P. Toennies and A. F. Vilesov, Annu. Rev. Phys. Chem., 1998, 49, 1. 2 J. P. Toennies and A. F. Vilesov, Angew. Chem., Int. Ed., 2004, 43, 2622.

3 F. Stienkemeier and K. K. Lehmann, J. Phys. B: At. Mol. Opt. Phys., 2006, 39, R127.

4 F. Stienkemeier and A. F. Vilesov, J. Chem. Phys., 2001, 115, 10119.

5 E. Loginov, D. Rossi and M. Drabbels, Phys. Rev. Lett., 2005, 95, 163401 .
6 A. Przystawik, P. Radcliffe, T. Diederich, T. Doppner, J. Tiggesbaumker and K. H. Meiwes-Broer, J. Chem. Phys., 2007, 126, 184306.

7 P. Radcliffe, A. Przystawik, T. Diederich, T. Doppner, J. Tiggesbaumker and K. H. Meiwes-Broer, Phys. Rev. Lett., 2004, 92, 173403.

8 S. Curtis, A. Boatwright, R. R. Wright and A. J. Stace, Chem. Phys. Lett., 2005, 401, 254.

9 E. Polyakova, D. Stolyarov and C. Wittig, J. Chem. Phys., 2006, 124.

10 R. Frochtenicht, J. P. Toennies and A. Vilesov, Chem. Phys. Lett., 1994, 229, 1.

11 S. Goyal, D. L. Schutt and G. Scoles, Phys. Rev. Lett., 1992, 69, 933.

12 P. Carcabal, R. Schmied, K. K. Lehmann and G. Scoles, J. Chem. Phys., 2004, 120, 6792.

13 M. Hartmann, F. Mielke, J. P. Toennies, A. F. Vilesov and G. Benedek, Phys. Rev. Lett., 1996, 76, 4560.

14 A. Braun and M. Drabbels, J. Chem. Phys., 2007, 127, 114305.

15 M. Hartmann, A. Lindinger, J. P. Toennies and A. F. Vilesov, J. Phys. Chem. A, 2001, 105, 6369.

16 D. M. Brink and S. Stringari, Z. Phys. D, 1990, 15, 257.

17 F. Dalfovo, A. Lastri, L. Pricaupenko, S. Stringari and J. Treiner, Phys. Rev. B, 1995, 52, 1193.

18 M. Lewerenz, B. Schilling and J. P. Toennies, Chem. Phys. Lett., 1993, 206, 381.

19 L. V. Keldysh, JETP, 1965, 20, 1307.

20 R. J. Levis and M. J. DeWitt, J. Phys. Chem. A, 1999, 103 6493.

21 A. Braun and M. Drabbels, J. Chem. Phys., 2007, 127, 114303.

22 E. Loginov and M. Drabbels, J. Phys. Chem. A, 2007, 111, 7504.

23 J. Harms, J. P. Toennies and F. Dalfovo, Phys. Rev. B, 1998, 58, 3341.

24 E. Knuth, B. Schilling and J. P. Toennies, Proceedings of the 19th International Symposium on Rarefied Gas Dynamics, 1995.

25 S. Stringari and J. Treiner, J. Chem. Phys., 1987, 87, 5021.

26 R. Schmied, P. Carcabal, A. M. Dokter, V. P. A. Lonij, K. K. Lehmann and G. Scoles, J. Chem. Phys., 2004, 121, 2701.

27 T. A. Stephenson, P. L. Radloff and S. A. Rice, J. Chem. Phys., 1984, 81, 1060.

28 A. Braun and M. Drabbels, J. Chem. Phys., 2007, 127, 114304.

29 M. N. Slipchenko, S. Kuma, T. Momose and A. F. Vilesov, Rev. Sci. Instrum., 2002, 73, 3600.

30 S. F. Yang, S. M. Brereton and A. M. Ellis, Rev. Sci. Instrum., 2005, 76, 104102.

31 C. S. Parmenter and M. W. Schuyler, J. Chem. Phys., 1970, 52, 5366.

32 S. Sharpe and P. Johnson, J. Chem. Phys., 1984, 81, 4176.

33 J. T. Graham and W. Weltner, J. Phys. Chem. A, 1999, 103 4594.

34 R. G. Neuhauser, K. Siglow and H. J. Neusser, J. Chem. Phys., 1997, 106, 896.

35 H. Buchenau, E. L. Knuth, J. Northby, J. P. Toennies and C. Winkler, J. Chem. Phys., 1990, 92, 6875.

36 H. Buchenau, J. P. Toennies and J. A. Northby, J. Chem. Phys., $1991,95,8134$.

37 A. Slenczka, B. Dick, M. Hartmann and J. P. Toennies, J. Chem. Phys., 2001, 115, 10199.

38 B. Dick and A. Slenczka, J. Chem. Phys., 2001, 115, 10206.

39 M. Rossi, M. Verona, D. E. Galli and L. Reatto, Phys. Rev. B, 2004, 69, 212510.

40 W. K. Lewis, C. M. Lindsay, R. J. Bemish and R. E. Miller, J. Am. Chem. Soc., 2005, 127, 7235.

41 W. K. Lewis, R. J. Bemish and R. E. Miller, J. Chem. Phys., 2005, 123, 141103.

42 D. Bonhommeau, M. Lewerenz and N. Halberstadt, J. Chem. Phys., 2008, 128, 054302.

43 K. K. Lehmann, Mol. Phys., 1999, 97, 645. 